\title{
3-D mapping with ellipsometrically determined physical thickness/refractive index of spin coated sol-gel silica layer
}

\author{
S DAS, P PAL, S ROY, S CHAKRABOARTY ${ }^{\dagger}$ and P K BISWAS* \\ Sol-Gel Division, ${ }^{\dagger}$ Computer Section, Central Glass and Ceramic Research Institute, Kolkata 700 032, India
}

\begin{abstract}
Precursor sol for sol-gel silica layer was prepared from the starting material, tetraethylorthosilicate (TEOS). The sol was deposited onto borosilicate crown (BSC) glass by the spinning technique (rpm 2500). The gel layer thus formed transformed to oxide layer on heating to $450^{\circ} \mathrm{C}$ for $\sim 30 \mathrm{~min}$. The physical thickness and the refractive index of the layer were measured ellipsometrically (Rudolph Auto EL II) at $632 \cdot 8 \mathrm{~nm}$. About $10 \times 10 \mathrm{~mm}$ surface area of the silica layer was chosen for evaluation of thickness and refractive index values at different points $(121$ nos.) with $1 \mathrm{~mm}$ gap between two points. Those data were utilized in the Autolisp programme for 3-D mapping. Radial distribution of the evaluated values was also displayed.
\end{abstract}

Keywords. Sol-gel silica layer; ellipsometric studies; refractive index; physical thickness; 3D-mapping.

\section{Introduction}

Spin coating usually develops a radial variation of thickness (Knittl 1976) and this is dependent on so many physical parameters such as dimension of substrate, viscosity and surface tension of precursor sol, boiling points of the solvent medium, vapour pressures of the used solvents and also of the resulting precursor sols, coating application rates, spinning time, etc. Maintaining all the conditions constant except the spinning rate, the radial variation may be minimized if the spinning rate be increased. Refractive index of the film may also be varied according to densification of the film which is dependent on the thickness. Hence, to optimize the coating application rate for a feasible homogeneous thickness, surface profiles for thickness and refractive index of deposited layer should be studied. In this regard, optical constants such as refractive index and thickness of the films were determined ellipsometrically $(\lambda=632.8 \mathrm{~nm})$ at different points with $1 \mathrm{~mm}$ interval of the surface of area $(10 \times 10 \mathrm{~mm})$. Altogether 121 points were selected with $1 \mathrm{~mm}$ gap in the $\mathrm{X}$ - and also in the Y-directions (i.e. $11 \times 11$ elements). Surface profiles with respect to the evaluated thickness and refractive index values were obtained in 3-D feature.

\section{Experimental}

\subsection{Preparation of sol}

The precursor of silica coating was prepared from acid hydrolysis of tetraethyl orthosilicate in a mixture of

*Author for correspondence 1-propanol and 2-butanol solvents. Concentration of the sol was 6 wt\% equivalent $\mathrm{SiO}_{2}$ (Biswas et al 1989).

\subsection{Deposition of coating}

Optically polished ( $\lambda$ order) borosilicate crown glass substrates of dimensions, $50 \mathrm{~mm}$ diameter and $6 \mathrm{~mm}$ thickness were chosen for coating. The substrates were cleaned ultrasonically and utilized for coating by the spinning method in the rpm range, 1500-5000. The coatings were cured at $450 \pm 5^{\circ} \mathrm{C}$ in an electrically heated furnace with a soaking of about $30 \mathrm{~min}$.

One of the samples which was coated with $2500 \mathrm{rpm}$ was chosen for the determination of thickness and refractive indices at 121 points in the area $10 \times 10 \mathrm{~mm}$ ellipsometrically (Rudolph Auto EL-II; source, He-Ne laser; wavelength, $632 \cdot 8 \mathrm{~nm}$ ) as this rpm develops oxide layer of about desired thickness $(\sim 1550 \AA)$ effective for the generation of antireflection (AR) coatings at around $1060 \mathrm{~nm}$ (Biswas et al 1999), a very important laser wavelength usually used for different applications.

\section{Characterization}

The diameter of the selected coated surface was $50 \mathrm{~mm}$. The central zone of about $10 \times 10 \mathrm{~mm}$ dimensions were chosen for having thickness and refractive index profile by the measurement of thickness (accuracy, $\pm 3 \AA$ ) and refractive index (accuracy, \pm 0.003 ) ellipsometrically at different points by shifting. The beam diameter of the $\mathrm{He}-\mathrm{Ne}$ laser of Rudolph ellipsometer was $25 \mu \mathrm{m}$. After measuring thickness at a point, say central point, $(x, y)=(0,0)$ the sample holder was shifted (Sasaki et al 1999 ) by an interval, $d_{x}$ (say, $1 \mathrm{~mm}$ ) in the direction of 
$\mathrm{X}$-axis to measure again thickness at $x+d_{x}, y$. In a similar way, thickness at $x, y+d_{y}$ was measured by shifting the holder in the direction of Y-axis. Thus, altogether 121 points were developed along the $\mathrm{X}$ - and $\mathrm{Y}$-directions

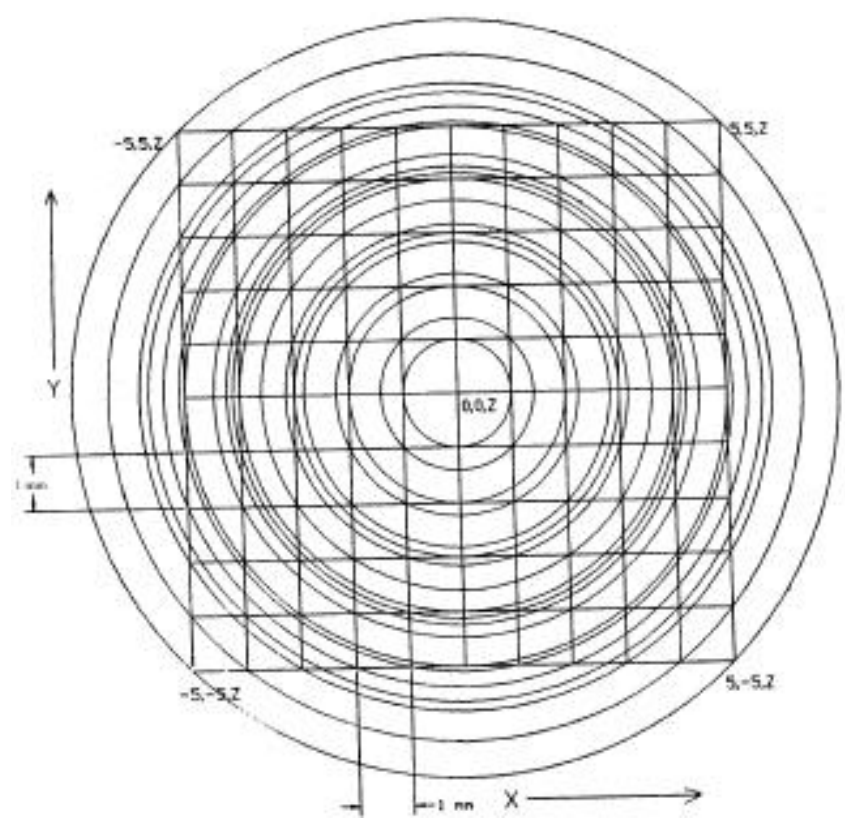

Figure 1. Distribution of $11 \times 11$ elements in $x-y$ plane and the concentric circles passing through the admissible elements intersecting $x-y$ lines. with $1 \mathrm{~mm}$ intervals. The points were monitored by sliding in-built screw-gauge fitted base plate used as sample holder.

To present 3-dimensional thickness and refractive index profiles, the corresponding values measured at different position coordinates were fed into in-house developed software. It consists of two modules. The first one is coded in Q-basic of Microsoft Corporation, which selects area for enlarged zoom. The second module, coded in Autolisp of M/s Autodesk Inc. produces 3-D view of the profile with thickness values along $\mathrm{Z}$-axis. Views at different view angles can also be obtained by utilizing this module. To represent actual variation in the optical parameters, any curve-fitting algorithm was not considered here. The adjacent points in the plots were joined with straight lines and to have more clear view surfing was also followed for 3-dimension surface feature.

The corresponding refractive index values of $R(x, y)$ with respect to $\mathrm{X}$-axis and $\mathrm{Y}$-axis were also used in the same programme to obtain surface profile with respect to refractive index.

\section{Results and discussion}

For surface profiles we used $11 \times 11$ elements followed by surfing to have clear surface feature. The 121 points $(11 \times 11)$ at the intersections between vertical and horizontal lines are depicted in figure 1. Thickness and

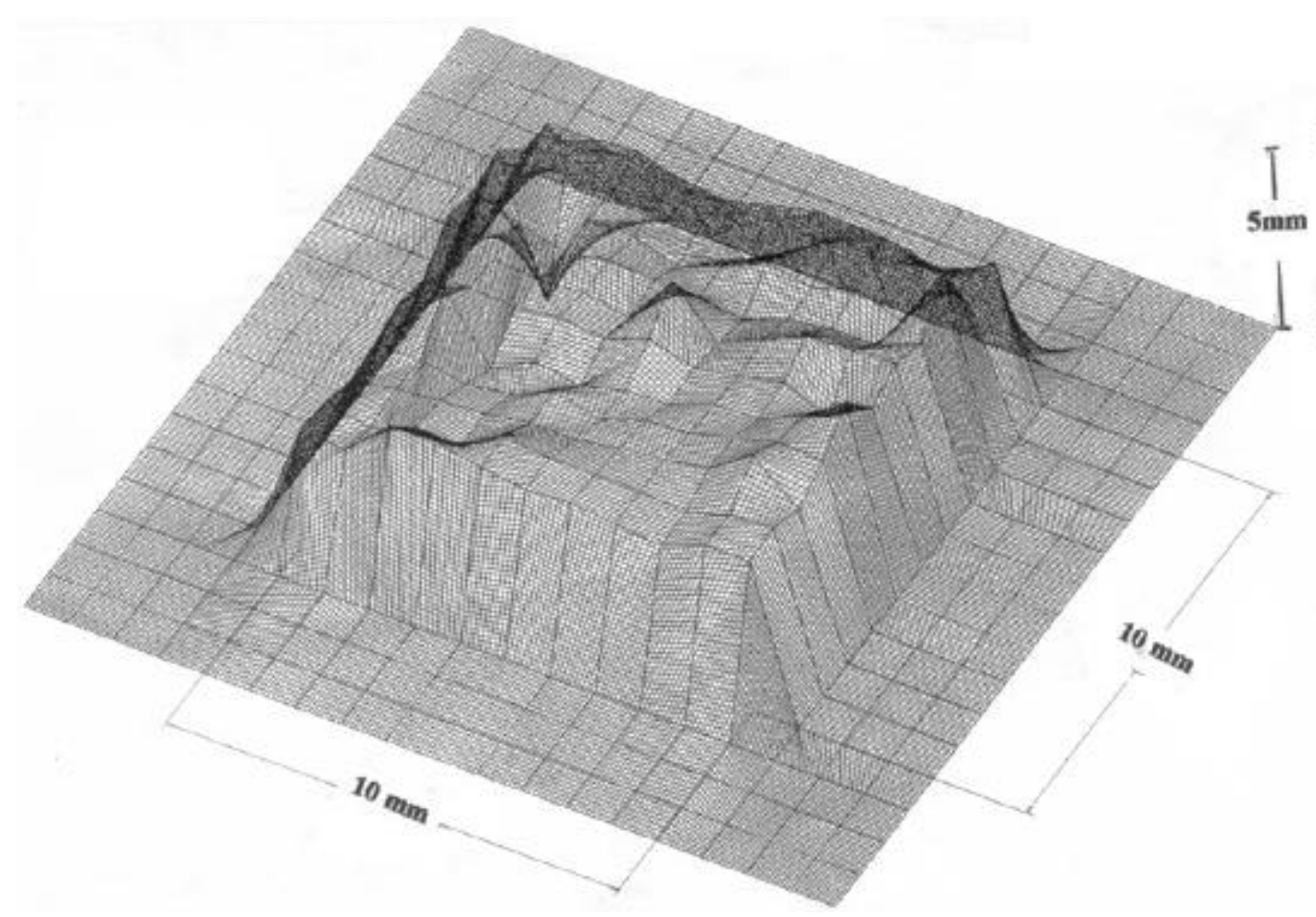

$1603 \AA$

Figure 2. Surface profile with respect to thickness $(5 \mathrm{~mm}=203 \AA)$. 


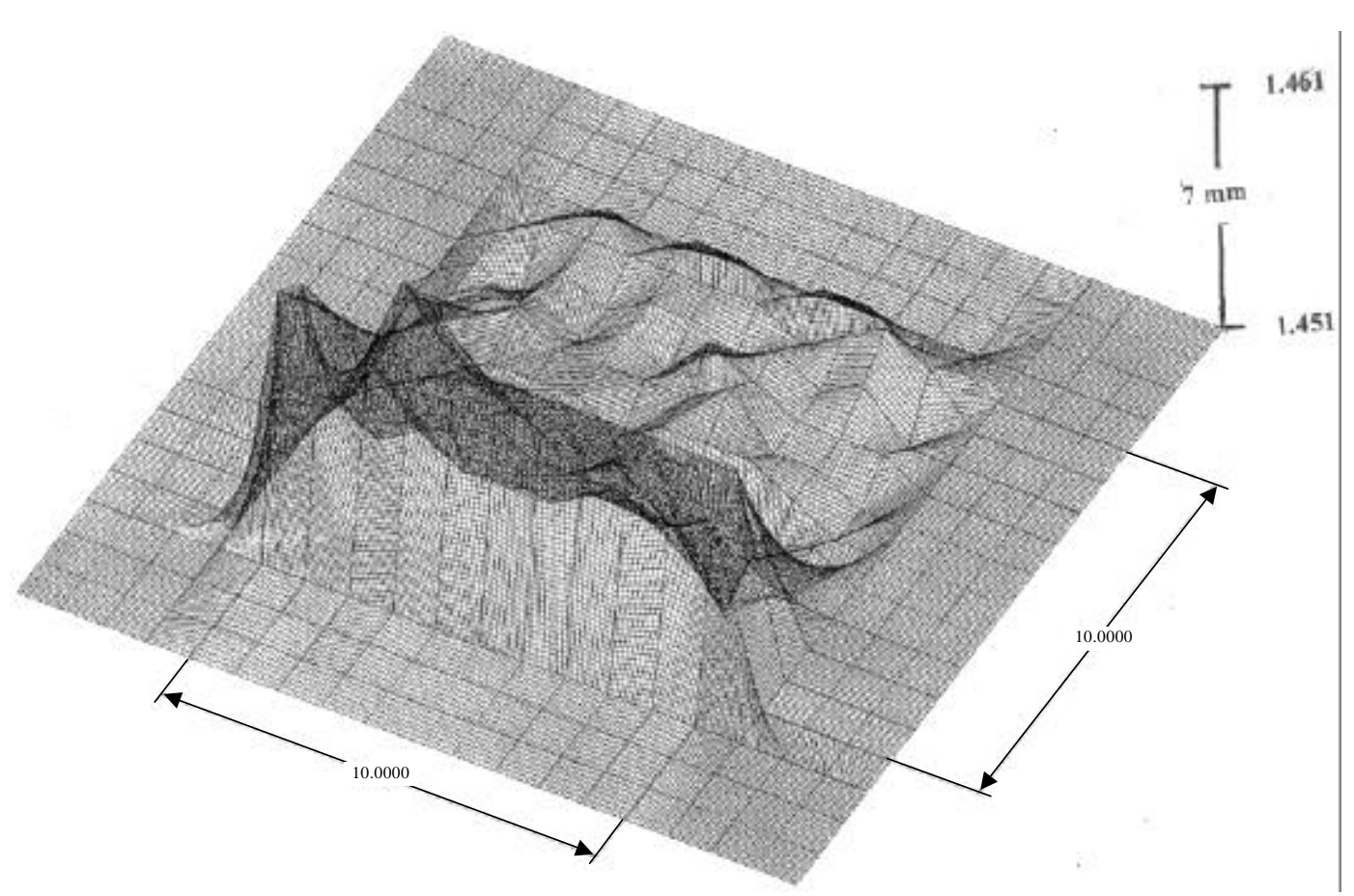

Figure 3. Surface profile with respect to refractive index $(5 \mathrm{~mm}=0 \cdot 010)$.

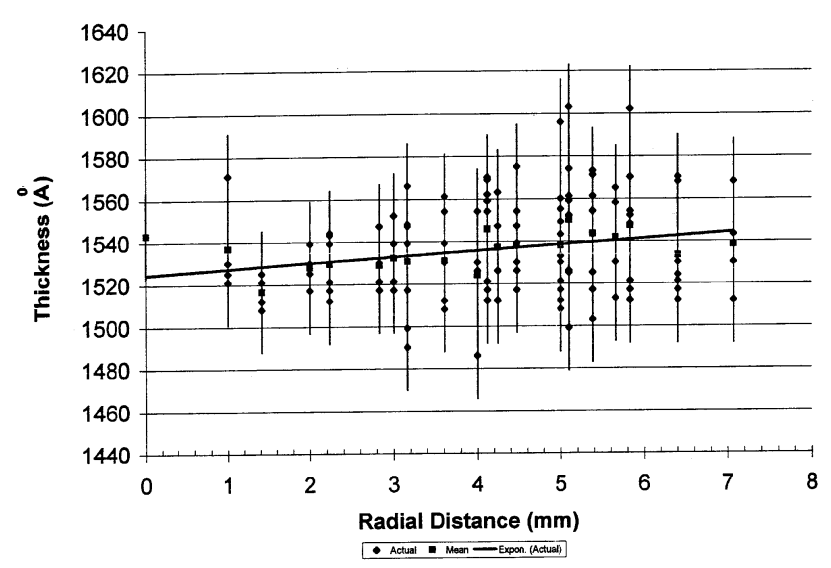

Figure 4. Plot of thickness vs radial distance of the elements passing through concentric circles as in figure 1 .

refractive index at those points were measured. Intervals of the measuring points were $1 \mathrm{~mm}$ and $1 \mathrm{~mm}$ along the $\mathrm{X}$ - and $\mathrm{Y}$-axes, respectively. After detecting central point as $(x, y)=(0,0)$ we detected $\left(x+d_{x}, y\right)$ and $\left(x, y+d_{y}\right)$ by giving shifts of $d_{x}$ and $d_{y}$ to the sample as the direction of polarized laser beam is fixed. The values of $d_{x}$ and $d_{y}$ were $1 \mathrm{~mm}$ and $1 \mathrm{~mm}$, respectively. We obtained thickness profile (not shown here) by computing thickness data along $Z$-axis in Autolisp programme. Similarly we obtained the refractive index profile (not shown here) for the corresponding refractive index values. When the values of $d_{x}$ and $d_{y}$ were minimized numerically to

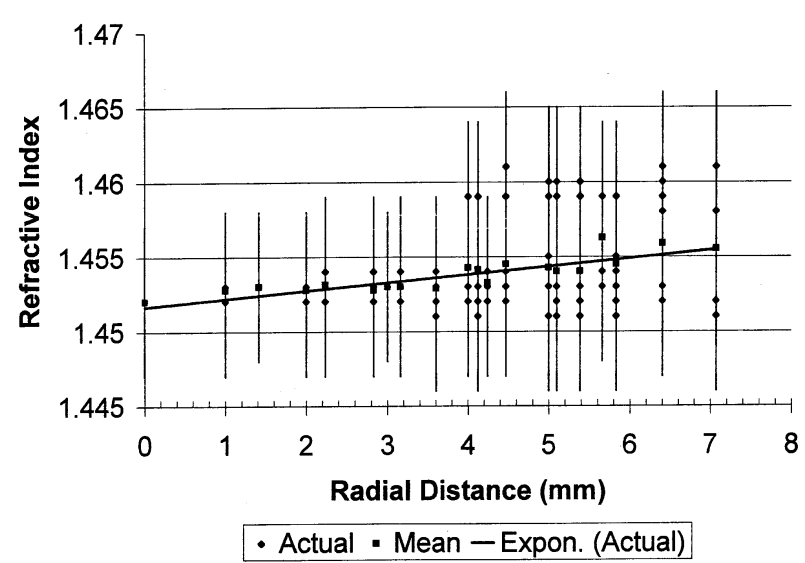

Figure 5. Plot of refractive index vs radial distance of the elements passing through concentric circles as in figure 1.

$0.05 \mathrm{~mm}$ and $0.05 \mathrm{~mm}$, respectively by surfing method, the surface features for thickness and RI profiles are distinct (figures 2 and 3). The edge region of figures 2 and 3 seemed rather steep than the central zone indicating relatively high thickness towards periphery. This was possibly due to the trend of decreasing centrifugal force towards periphery.

To understand radial distribution of the deposited materials a number of concentric circles were drawn on the measured plane on the basis that these should pass through maximum number of intersecting points (figure 1). The thickness data of the equidistant points from the 
centre $(x, y=0,0)$ were plotted against radial distance (figure 4). The mean of the points exhibited trend of increasing thickness radially and it is maximum at the periphery. This trend may be minimized if the coating application rate be increased. The detailed work on this is in progress. The variation of thickness of the concentric points is possibly due to the development of striation on the surface which basically forms a surface of periodic high and low thickness.

Similarly the refractive index data obtained from the concentric points were also plotted (figure 5) against radial distance. This also exhibited similar feature.

\section{Conclusion}

Surface profile (with respect to thickness and refractive index measured ellipsometrically) of silica layer of $10 \times 10 \mathrm{~mm}$ was obtained by 3 -D mapping using Autolisp programme. Altogether $11 \times 11$ (=121 points) elements were utilized for the mapping. Radial variation of the optical parameters due to spinning was also noted.

\section{Acknowledgement}

The authors acknowledge the sponsor, BRNS, DAE for financial assistance to carry out the work.

\section{References}

Biswas P K, Kundu D and Ganguli D 1989 J. Mater. Sci. Lett. 8 1436

Biswas P K, Sujatha-Devi P, Chakraborty P K, Chatterjee M, Kamath M P, Joshi A S and Ganguli D 1999 in Laser materials and devices (eds) A Mallik and R R Dayal (New Delhi: Allied Publishers Ltd.) p. 314

Knittl Z 1976 Optics of thin films (London: John Wiley \& Sons)

Sasaki O, Nakada T and Suzuki T 1999 Opt. Engg. 381679 